\title{
A preliminary study on parasympathetic-sympathetic interaction through the analysis of heart rate variability and electrodermal activity
}

\author{
Alejandro Luis Callara ${ }^{1}$, Laura Sebastiani ${ }^{2}$, Enrica Laura Santarcangelo ${ }^{2}$, Shadi Ghiasi ${ }^{1}$, Elisabetta Fratini ${ }^{1}$, \\ Antonio Lanata ${ }^{1}$, Nicola Vanello ${ }^{1}$, Enzo Pasquale Scilingo ${ }^{1}$, and Alberto Greco*1,
}

\begin{abstract}
In this preliminary study, we propose to investigate the interaction between the parasympathetic (PNS) and the sympathetic nervous system (SNS) during an isometric handgrip task. SNS and PNS directed coupling was estimated by applying a time-varying bivariate autoregressive model to spectral features extracted from the heart rate variability and the electrodermal activity signals. Significant changes in the PNS-SNS interactions were observed in both directions and between rest and handgrip conditions.
\end{abstract}

\section{INTRODUCTION}

The autonomic nervous system (ANS) is the primary mechanism to unconsciously regulate most of the bodily functions such as heart rate variability (HRV), respiratory rate, electrodermal activity (EDA), urination, and digestion [1]. The two branches of the ANS, i.e., the parasympathetic (PNS) and the sympathetic nervous systems (SNS), are generally recognized to exert antagonistic effects on the regulation of autonomic functions. However, this opposing interplay is not algebraically additive, but complicated interactions exist [2]. Indeed, plenty of experimental and clinical studies have demonstrated the presence of multiple interactions between PNS and SNS that are mediated through several pathways and mechanisms at both central and peripheral levels [3].

In this preliminary study, we aim at characterizing the directional interdependence between the PNS and SNS through the analysis of two widely used ANS correlates such as the HRV and the EDA. In fact, the estimation of the high frequency (HF) components of the HRV spectrum is commonly considered a reliable measure of the PNS activity on cardiac functioning [4]. The spectral power of the EDA, in the range of 0.045 to $0.25 \mathrm{~Hz}$ (EDASYMP), has been recently presented as an index of the SNS activity under cognitive, orthostatic and physical stress (handgrip) conditions [5]. To detect and quantify the bilateral interactions between the two branches of the ANS, we adopted a bivariate autoregressive (BVAR) model [6]. Due to the time-varying relationships between these signals, we applied a modified recursive Kalman filter to track model parameter changes.

\footnotetext{
This work was not supported by any organization

1 ALC, SG, EF, AL, NV, EPS, and AG, are with the Dipartimento di Ingegneria dell'Informazione, University of Pisa, Largo Lucio Lazzarino, 1, 56122, Pisa, Italy.

${ }^{2}$ LS and ELS are with the Department of Physiology and Biochemistry "G. Moruzzi", University of Pisa, Via S. Zeno 31, 56127 Pisa, Italy

*Corresponding author email: alberto.greco@unipi.it

978-1-7281-5751-1/20/\$31.00 (C)2020 IEEE
}

\section{Materials And Methods}

Twenty-five healthy subjects (aged $24 \pm 2$ years) underwent an isometric handgrip test. The protocol consisted of 3 min of resting-state (rest0) and 2 min during which the subject was asked to tighten a small hard ball in his/her dominant hand at the maximum contraction strength (hg). The hg session was considered divided into two halves (hg1 and hg2). The ECG and EDA signals were recorded using the BIOPAC MP 150 system with a sampling frequency of $500 \mathrm{~Hz}$.

EDA signal represents changes in the skin conductance of the non-dominant hand due sweat gland activity. Since sweat glands are innervated by the SNS, EDA is considered an ideal way to estimate the SNS activity. ECG signals were used to detect R-peaks in order to generate RR time series that were subsequently resampled at $4 \mathrm{~Hz}$ (HRV). The EDA signals were also resampled at $4 \mathrm{~Hz}$.

From HRV and EDA, we estimated the HF power spectrum and EDASYMP, respectively. More in detail, the timefrequency representation of both the HRV and EDA signals were calculated using the smoothed pseudo-WignerVille distribution method (SPWVD) [7]. SPWVD allowed to achieve a better time-frequency resolution with respect to non-parametric linear methods [8].

\section{A. Parasympathetic-sympathetic causal interaction}

For each subject, the HF and EDASYMP time-series were used to construct bivariate autoregressive models from which the HF-EDASYMP bilateral interactions were explored. In particular, we exploited the framework presented in [6] to estimate time-varying (TV-) model coefficients based on an optimized kalman-filter approach. Furthermore, the heteroskedasticity of model residuals was evaluated in order to take into account possible TV- model residual variances. Indeed, this represents a major issue in the estimation of coupling measures obtained from TV-BVAR models, leading to inaccuracies in both strength and directionality of coupling estimates. To properly compare the amount of interaction across different subjects we estimated the generalized Partial Directed Coherence (gPDC) [9], [10] starting from the TVmodel coefficients and covariances. This results in a scaleinvariant estimator of granger-causal interactions between different time-series. The statistical significance of observed gPDC was assessed through a phase-randomization approach [11]. Group level analysis was performed by averaging surrogates distributions from each subject. The averaging was performed for each direction of interaction, each frequency, 


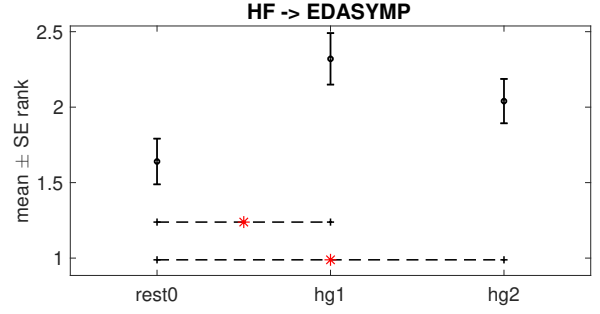

Fig. 1. Within-subject ranks of the HF $\rightarrow$ EDASYMP index obtained for the three sessions. Statistical pairwise comparisons found significant differences in the cases indicated by the asterisks.

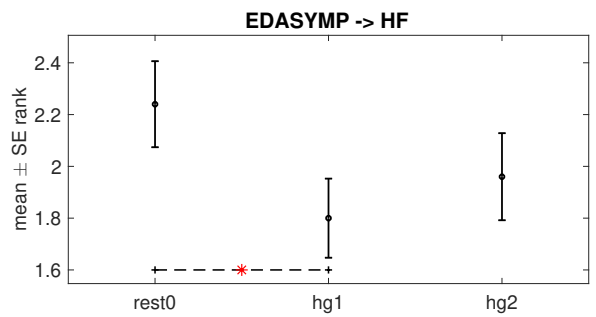

Fig. 2. Within-subject ranks of the EDASYMP $\rightarrow$ HF index obtained for the three sessions. Statistical pairwise comparisons found significant differences in the cases indicated by the asterisks.

and each time-window, i.e for each $(i, j, \omega, t)$. Statistically significant causality was obtained by comparing average gPDC across subjects with the group-level null-distribution. Finally, an inter-subject Wilcoxon test was used to compare gPDC values among the resting session and the first and second half of the handgrip session. The analysis was performed for both HF $\rightarrow$ EDASYMP and EDASYMP $\rightarrow$ HF directions. In all statistical tests, false discovery rate was controlled through the Benjamini-Hockberg-Yekuteli correction for multiple testing.

\section{RESUlts}

The statistical analysis through surrogate data evidenced significant interactions between HF and EDASYMP. Particularly, we observed that during both REST and HG conditions, $\mathrm{HF} \rightarrow$ EDASYMP and EDASYMP $\rightarrow$ HF connections significantly differed from 0 according to the phase-randomization surrogate testing ( $p<0.05$, FDR-corrected). Moreover, the directionality of causal interactions changed according to the experimental condition (Fig. 1 and 2). Specifically, we observed a significant increase $(p<0.05)$ in the flow of information going from HF to EDASYMP during the HG task with respect to the REST condition (Fig. 1). On the other hand a decrease of the EDASYMP to HF interaction was observed during the first half of the HG task (Fig. 2).

\section{Discussion AND CONCLUSION}

In this preliminary study, we propose a novel approach to investigate the interaction between the PNS and SNS during a handgrip task. The activities of the PNS (HF) and SNS (EDASYMP) were estimated through the spectral analysis of the HRV and the EDA, respectively, by using the SPWD method for a better time resolution. Our results highlighted a dynamic interaction between HF and EDASYMP, that could reflect a communication between the PNS and SNS. More in detail, the TV-MVAR model revealed a significant increase of information flow from HF to EDASYMP during the whole handgrip task. Contrarily, the information flow from EDASYMP to HF significantly decreased at the beginning of $\mathrm{HG}$ and then recovered to the resting level during the second half of the task.

It is worthwhile noting that the PNS is commonly responsible for the regulation of activities occurring at rest, whereas the SNS is responsible for the flight-or-fight response such as the one arisen by the handgrip task. Interestingly, our preliminary results may suggest that the flow of information is driven by the ANS branch which is not principally involved in the regulation of such a physiological condition.

It is important to underline that the observed influences, although informative, might not be true causal relationships between PNS and SNS. This hypothesis should be confirmed by using a richer description of SNS and PNS activities (i.e. by using more signals) and by better characterizing their manifestation throughout the estimated indices. Future works will try to solve these open questions. In addition, further analyses will focus on the time-frequency characterization of the interaction and on the application of the proposed method to new experimental protocols that may involve orthostatic and emotional stimuli.

\section{REFERENCES}

[1] L. K. McCorry, "Physiology of the autonomic nervous system," American journal of pharmaceutical education, vol. 71, no. 4, p. 78, 2007.

[2] M. N. Levy, "Brief reviews: sympathetic-parasympathetic interactions in the heart," Circulation research, vol. 29, no. 5, pp. 437-445, 1971.

[3] K. Ondicova and B. Mravec, "Multilevel interactions between the sympathetic and parasympathetic nervous systems: a minireview," Endocr Regul, vol. 44, no. 2, pp. 69-75, 2010.

[4] M. V. Kamath and E. L. Fallen, "Power spectral analysis of heart rate variability: a noninvasive signature of cardiac autonomic function." Critical reviews in biomedical engineering, vol. 21, no. 3, pp. 245311, 1993.

[5] H. F. Posada-Quintero, J. P. Florian, A. D. Orjuela-Cañón, T. AljamaCorrales, S. Charleston-Villalobos, and K. H. Chon, "Power spectral density analysis of electrodermal activity for sympathetic function assessment," Annals of biomedical engineering, vol. 44, no. 10, pp. 3124-3135, 2016

[6] K. Kostoglou, A. D. Robertson, B. MacIntosh, and G. D. Mitsis, "A novel framework for estimating time-varying multivariate autoregressive models and application to cardiovascular responses to acute exercise," IEEE Transactions on Biomedical Engineering, 2019.

[7] M. Orini, R. Bailón, L. T. Mainardi, P. Laguna, and P. Flandrin, "Characterization of dynamic interactions between cardiovascular signals by time-frequency coherence," IEEE Trans. on Biom. Eng., vol. 59, no. 3, pp. 663-673, 2012.

[8] S. Pola, A. Macerata, M. Emdin, and C. Marchesi, "Estimation of the power spectral density in nonstationary cardiovascular time series: assessing the role of the time-frequency representations (tfr)," IEEE Transactions on Biomedical Engineering, vol. 43, no. 1, p. 46, 1996.

[9] L. A. Baccala, K. Sameshima, and D. Y. Takahashi, "Generalized Partial Directed Coherence," in 2007 15th International Conference on Digital Signal Processing, Jul. 2007, pp. 163-166, iSSN: 15461874, 2165-3577.

[10] J. R. Sato, D. Y. Takahashi, S. M. Arcuri, K. Sameshima, P. A. Morettin, and L. A. Baccalá, "Frequency domain connectivity identification: An application of partial directed coherence in fMRI," Human Brain Mapping, vol. 30, no. 2, pp. 452-461, Feb. 2009.

[11] J. Theiler, S. Eubank, A. Longtin, B. Galdrikian, and J. Doyne Farmer, "Testing for nonlinearity in time series: the method of surrogate data," Physica D: Nonlinear Phenomena, vol. 58, no. 1, pp. 77-94, Sep. 1992. 